\title{
A Constrained Shortest-Path Energy-Aware Routing Algorithm for Wireless Sensor Networks
}

\author{
Moustafa A. Youssef \\ Department of Computer Science \\ University of Maryland at College Park \\ A.V. William's Building \\ College Park, MD 20742
}

\author{
Mohamed F. Younis* \\ Dept. of Comp. Sc. and Elect. Eng. \\ University of Maryland Baltimore County \\ 1000 Hilltop Circle \\ Baltimore, MD 21250
}

\author{
Khaled A. Arisha \\ Honeywell Intl. Inc. \\ Advanced Systems Tech. Group \\ 7000 Columbia Gateway Drive \\ Columbia, MD 21046
}

\begin{abstract}
While traditional routing protocols try to minimize the end-to-end delay or maximize the throughput, most energyaware routing protocols for wireless sensor networks try to extend the life time of the network by minimizing the energy consumption sacrificing other performance metrics. In this paper, we introduce a new energy-aware routing protocol that tries to minimize the energy consumption and, at the same time, maintain good end-to-end delay and throughput performance. The new algorithm is based on a constrained shortest-path algorithm. We compare the new algorithm with some traditional routing and energy-aware routing algorithms. The results show that the new algorithm performance is acceptable under all performance metrics and presents a performance balance between the traditional routing algorithms and the energy-aware routing algorithms. The constraint value can be chosen to achieve different performance objectives for different sensor network missions.
\end{abstract}

\section{INTRODUCTION}

Sensor networks can enable "smart environments" which can monitor ambient conditions such as temperature, movement, sound, light, location and others. Wireless sensor network technology poses its unique design challenges. One important feature that distinguishes sensor networks from traditional distributed systems is their need for energy efficiency. Many nodes in the emerging sensor systems will have finite energy reserves from a battery. The scale of a sensor network's deployment will make recharging these energy reserves impossible. Although energy efficiency can be improved at various layers of the communication protocol stack, most published research has focused on hardware-related energy efficiency aspects of wireless communications. Low-power electronics, power-down modes, and energy efficient modulation are examples of work in this category [1] However, due to fundamental physical limitations, progress towards further energy efficiency is expected to be achieved in other layers.

Energy-aware routing has started to receive attention in the recent few years. While traditional routing protocols in wired networks emphasize on maximizing end-to-end throughput and minimizing delay, energy constraints have become a central issue in wireless networks. Many energy-aware routing metrics have been proposed, e.g. [2] 4], to minimize the energy consumption and increase the network lifetime. A number of energy-aware routing protocols have been introduced, as in [2][7]. However, some applications, such as target tracking in a military environment, are delay sensitive and an algorithm that tries to optimize the energy consumption alone may lead to unacceptable end-to-end delay. In [8] we introduced a new energy-aware routing algorithm that uses a multi-objective cost function to balance the energy efficiency requirements with other quality of service requirements. In this paper, we handle the problem of balancing the energy efficiency and other performance metrics using a different approach. We introduce a new energy-aware routing protocol for wireless sensor networks that uses the distance as a measure for transmission energy consumption and for estimating the propagation delay. By changing the network topology graph using constraints on the maximum transmission distance for each sensor node, the algorithm can be tailored to achieve good values for energy consumption, end-to-end delay and throughput. We study the performance of the new algorithm by comparing it with traditional routing algorithms, which try to maximize the throughput or minimize the end-to-end delay, and with other energy-aware algorithms.

We assume that the sensor network is organized into clusters. Our focus in this paper is on routing within the cluster. Every cluster has a gateway node that is responsible for the mission-oriented organization of the sensors by determining the set of sensors that will be responsible for sensing the environment. The gateway receives sensed data from the sensors and sends reports generated through data fusion of sensor readings to a command node. The gateway node is assumed to be located within the communication range of all the sensors of its cluster. Sensors are equipped with a short-range radio transceiver, and are responsible for detecting ambient conditions. The command node will inform each gateway node of the ID and location of sensors allocated to the cluster. Gateway nodes, which are significantly less energy-constrained than the sensors, interface the command node with the sensor network via long-haul communication links.

Sensors are assumed to be capable of operating in an active mode or a low-power stand-by mode. The sensing and processing circuits can be powered on and off. In addition, both the radio transmitter and receiver can be turned on and off independently and the transmission power can be programmed based on the required range. Thus, nodes in a cluster can be in one of four main states: sensing only, relaying only, sensing-relaying, and inactive. In the sensing state, the node's sensing circuitry is on and it sends data to the gateway in a constant rate. In the relaying state, the node does not sense the target but its communications circuitry is on to relay the data from other active nodes. When a node is both sensing the target and relaying messages from other nodes, it is considered in the sensing-relaying state. Otherwise, the node is considered an inactive node and can turn off its sensing and communication circuitry. The decision of determining the node's state is done at the gateway based on the current target position, node battery levels,

\footnotetext{
${ }^{*}$ This work was done while the author was at Honeywell International Inc..
} 


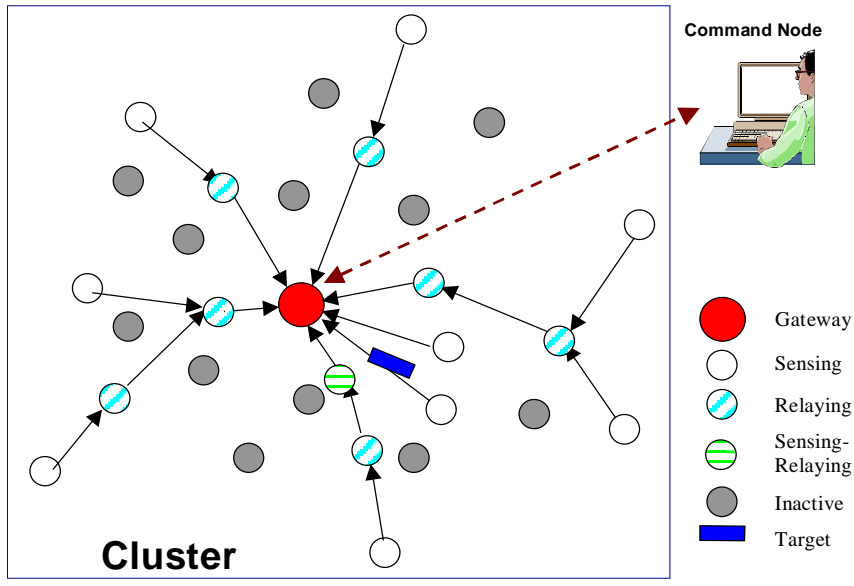

Fig. 1: A Typical Cluster in a Sensor Network

and desired network performance measures. Fig. 1 shows a typical cluster tasked with a target-tracking mission.

The paper is organized as follows: the next section describes the system model and the new algorithm in details. Section III presents the simulation environment and the performance analysis. Finally, Section IV concludes the paper and gives directions for future work.

\section{PROTOCOL DESCRIPTION}

Usually routes are represented in a table that specifies the path of messages between two nodes. Setting routes for sensor data can be performed in a central node that knows the network topology, e.g. the gateway, or distributed among the sensors themselves. Both centralized and distributed routing requires maintenance of the routing table every time the network topology changes. While distributed approaches are scalable for larger networks, updating routing tables and ensuring consistency among the local versions that the sensor nodes have consume significant computation and communication resources, thus limiting the portion of the already limited sensor's energy that can be dedicated to serve the application. In addition, exchanging routing messages among the sensors will create excessive traffic that drains unnecessary energy since radio receivers on the sensors may overhear the routing message transmissions not destined to them. Therefore, we choose to assign the routing decision within the cluster to the gateway.

Managing the routing decision centrally at the gateway can be seen as a logical extension to the gateway's role, specially as all sensor readings have to be forwarded to the gateway for fusion and application-specific processing. Moreover, centralized routing is simple and fits the nature of the sensor networks. Since the sensor is committed to data processing and communication, it is advantageous to offload routing decision from the resourceconstrained sensor nodes. In addition, since the gateway has a cluster-wide view of the network, the routing decisions should be more efficient than the decisions based on local views at the sensor level. Given that the gateway organizes the sensors in the cluster, it can combine the consideration for energy commitments to data processing, remaining sensor energy, sensors' location, and acceptable latency in receiving the data in efficiently setting message routes.

The typical operation of the network consists of two alternating cycles: data cycle and routing cycle. During the data cycle, the nodes sensing the target send their data to the gateway. During the routing cycle, the state of each node in the network is determined by the gateway and the nodes are then informed about their newly assigned states and how to route the data.

In the rest of this section we introduce the new routing algorithm, discuss when rerouting is performed, and give an overview of the energy-aware MAC layer protocol that we use.

\section{A. A Constrained Shortest-Path Routing Protocol}

In the new algorithm, we use the distance between any two nodes as an estimate for the transmission power required to send a packet between the two nodes and the propagation delay between them. The transmission energy required to send a bit has been found to have a distance dependence which is well modeled by $d^{n}$, where $d$ is the distance between the transmitter and the receiver antennas and the exponent $n$ is determined from field measurements and the particular system at hand [7][8]. Moreover, the propagation delay is directly proportional to the distance and related to it with the speed of signal propagation in the wireless medium.

By constraining the maximum transmission distance that the wireless transmitter of each sensor node can reach, we can change the interconnection between the nodes and thus obtain different network topologies. We model the routing problem as a single-sink unicast routing problem from the sensor nodes to the gateway taking our cost metric as the number of hops. This problem can be solved by any shortest-path routing algorithm.

It is important to mention here that without using a constraint on the maximum transmission distance, the minimum number of hops routing algorithm reduces to the direct routing algorithm, in which each node sends its data directly to the gateway, as we assume that all the nodes can reach the gateway directly using their wireless transceivers.

Fig. 2 shows an example of the effect of changing the maximum transmission distance constraints on the network topology. By choosing a large value for the maximum transmission distance constraint, as in Fig. 2.b, the network topology approaches a complete graph and the minimum number of hops routing algorithm becomes the direct routing algorithm. This has the effect of decreasing the end-to-end delay as each node sends its data directly without relaying or queuing delay. However, using direct transmission to the gateway increases the transmission energy consumption significantly especially for nodes far away from the gateway.

Choosing a small value for the maximum transmission distance constraint, as in Fig. 2.c, leads to sparse graph for the network topology in which each node is approximately connected to its nearest neighbor. This, in general, has the effect of decreasing the transmission power, as the distance is reduced, however, end-to-end delay is increased as multiple relays are used.

By choosing a suitable value for the maximum transmission distance constraint, the performance metrics obtained can be tailored to the desired level. This is studied in more details in Section III.

\section{B. Rerouting Decision}

For a target tracking sensor network, the selected sensors will vary as the target moves. Changes in the selection of active sensors will have to be accommodated by the routing algorithm in order to ensure that delivery of the sensed data and the proper tracking of the target. In addition the gateway will continuously monitor the available energy level at every sensor using a model-based energy consumption for the data processor, radio transmitter and receiver to track the life of the sensor battery [8]. This model is used to 


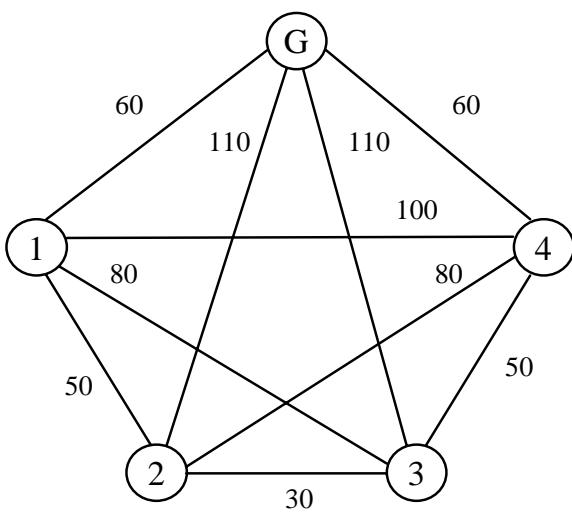

a. Distance Between Nodes

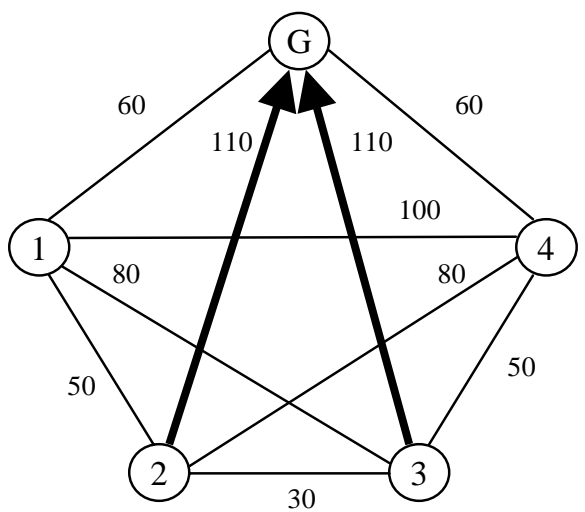

b. Maximum Transmission Distance $=125$

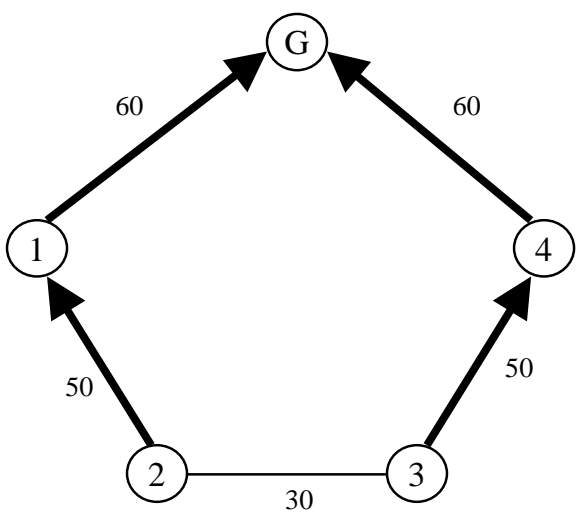

c. Maximum Transmission Distance $=75$

Fig. 2: An example of changing network topology by changing the maximum transmission distance (assuming nodes 2 and 3 are chosen to sense the environment.) (a) Distance between nodes. (b) Network topology and routes from the sensor nodes to the gateway using the minimum number of hops routing algorithm for maximum transmission distance $=125$. (c) Network topology and routes from the sensor nodes to the gateway using the minimum number of hops routing algorithm for maximum transmission distance $=75$.

determine when to perform rerouting. However, the model needs to be refreshed periodically to correct any deviation from the actual levels of the nodes' battery. Thus, the decision of the gateway to perform rerouting is based on the following three criteria:

1. Sensor reorganization: The gateway may perform rerouting if an event happens that requires the reselection of active sensors.

2. Nodes' Battery Energy Level: The gateway may perform rerouting if the battery level of any active node drops to a certain level.

3. Energy Model Adjustment: Rerouting can also occur after receiving an updated status from the sensors. Changes to the energy model might affect the optimality of the current routes, and thus new routes have to be generated.

\section{MAC Layer Protocol}

Although the proposed algorithm is independent of the MAC layer protocol, choosing an energy-aware MAC layer protocol may add to the energy efficiency. Existing MAC layer protocols can be divided into two groups: contention-based and contention-free. Contention-based techniques are very efficient when the network load is low, however, they cannot provide stability under heavy network loads [10] Moreover, stations prevent each other from taking control of the channel due to contention which makes the average delay increases rapidly and wastes the scarce energy resources. Therefore, we choose to implement a contention-free time division multiple access (TDMA) based MAC layer whose slot assignment is managed by the gateway. The gateway informs each node about slots in which it should listen to other nodes' transmission and slots that the node can use for its own transmission. The TDMA MAC layer provides two features that are advantageous to our approach. First, clock synchronization is built in the TDMA protocol. Second, collision among the nodes can be avoided with assigning non-overlapping time slots.

To set the routes, the gateway sends to each sensing node the transmission range to cover so that data can reach the next relay node on the route. In addition, the gateway sends relay nodes a forwarding table. The forwarding table consists of ordered tuple of the form: (time slot, data-originating node, transmission range). The "time slot" entry specifies when to turn the receiver on in order to listen for an incoming packet. The "source node" is the sensor node that originated this data packet, and the "transmission range" specifies the transmission power to use in sending the data. This transmission power should be enough to reach the next relay on the path from the originating node to the gateway. The transmission range ensures that the next relay node, which is also told to forward that data packet, can clearly receive the data packet.

The next section describes detailed performance evaluation of the energy-aware routing approach via simulation.

\section{PERformance Evaluation}

In this section, we use the simulation technique to study the effect of the maximum transmission distance constraint on the performance of the new algorithm. We further compare the performance of the new algorithm with two routing algorithms. In the first algorithm, - the direct routing algorithm- each node sends its data directly to the gateway. The direct routing algorithm is an example of algorithms that tries to minimize the end-to-end delay. In the second algorithm -minimum transmission energy routing algorithm- the cost function chosen tries to minimize the sum of the distance squared between the node and the gateway [4]. The minimum transmission energy routing algorithm is an example of the current energy-aware routing algorithms.

We start by listing the performance metrics we use to evaluate the new routing algorithm. Then, we present the simulation environment and the simulation results.

\section{A. Performance Metrics}

We use different performance metrics that capture different performance requirements. These metrics are:

- Time to network partition: Defined as the time for the first node to die in the network [2]

- $\quad$ Time for last node to die: This metric, along with the time to network partition metric, gives an indication of network lifetime. 
- $\quad$ Average and standard deviation of nodes' lifetime: This also gives a good measure of the network lifetime. A routing algorithm, which minimizes the standard deviation of nodes' life, is predictable and thus desirable.

- $\quad$ Average delay per packet: Defined as the average time a packet takes from a node in the sensing state to the gateway.

- Network Throughput: Defined as the rate of data packets received at the gateway.

- $\quad$ Average energy consumed per packet: This metric represents the average energy consumed in transmitting, and receiving a data packet. A routing algorithm that minimizes the energy consumed per packet will, in general, yields better energy savings and increased network lifetime.

\section{B. Environment Setup}

The cluster consists of 100 nodes placed randomly in a $1000 \times 1000$ meter square area. The gateway position is determined randomly within the cluster boundaries. A free space propagation channel model [11] is assumed with data rate set to $2 \mathrm{Mb} / \mathrm{s}$. Packet lengths are $10 \mathrm{kbit}$ for data packets and $2 \mathrm{kbit}$ for routing and refresh packets. The buffer size at each node is 15 packets. Each node has an initial energy of 2 joules. A node is considered non-functional if its energy level reaches zero.

For a node in the sensing state, packets are generated at a constant rate of one packet/second. This value is consistent with the specifications of the Acoustic Ballistic Module from SenTech Inc [12]. Each data packet is time-stamped when it is generated to allow the calculation of average delay per packet. In addition, each packet has an energy field that is updated during the packet transmission to calculate the average energy per packet. A packet drop probability is taken equal to 0.01 . This is used to make the simulator more realistic and to simulate the deviation of the gateway energy model from the actual energy model of nodes.

We assume that the cluster is tasked with a target-tracking mission. The initial set of sensing nodes is chosen to be the nodes on the convex hull of the sensors of the cluster. The set of sensing nodes changes as the targets move. Since targets are assumed to come from outside the cluster, the sensing circuitry of all boundary nodes is always turned on. The sensing circuitry of other nodes are usually turned off but can be turned on according to targets movement.

As mentioned before, rerouting occurs when a node's energy level falls below a percentage of its initial energy. This percentage is taken equal to $80 \%$. Each time this threshold is reached, it is reset to 0.8 of its previous value.

For energy-consumption, we used the communication energy consumption model used in [4][6], the computation energy consumption model used in [6]|13] and the sensing energyconsumption model used in [14]

Targets are assumed to start at a random position outside the convex hull. We experimented with different types of targets but for this paper we choose the linearly moving targets. These targets are characterized by having a constant speed chosen uniformly from the range four meters/second to six meters/second and a constant direction chosen uniformly depending on the initial target position in order for the target to cross the convex hull region. For the purpose of this experiment we assume that only one target will be active at any time. Each target remains active until it leaves the deployment region area. In this case, a new target is generated.

\section{Performance Results}

In this section, we study the effect of changing the maximum transmission distance constraint on the performance of the new algorithm. Then, we compare the performance of the new algorithm with the direct routing algorithm and the minimum transmission energy routing algorithm.

\section{1) Effect of Changing the Maximum Transmission Distance Constraint on Perfroamcne}

Figures 3 through 6 show the effect of changing the maximum transmission distance constraint on the performance of the new algorithm. Fig. 3 shows that as the maximum transmission distance constraint increases, the average energy consumed per packet increases. This is expected, as when the maximum transmission distance constraint increases the network topology graph becomes denser and the minimum number of hops routing algorithm chooses the long links to minimize the number of hops. These long links leads to more transmission energy consumption per packet. The same reasoning applies to figures 4 and 5. As the maximum transmission distance constraint increases, fewer number of hops in chosen by the minimum number of hops routing algorithm leading to decreased end-to-end delay. As the end-to-end delay is decreased, the throughput increases as more packets reach the gateway. As the average energy consumed per packet increases, the network lifetime parameters are decreased as shown in Fig. 6.

For low values for the maximum transmission distance constraint, the network topology graph is sparse and the length of the links is small leading to reduced average energy consumed per packet. However, the end-to-end delay is increased due to the increase of the number of relays between a sensor node and the gateway.

Form figures 3 through 6 we can see that a good value for the maximum transmission distance constraint should be between 200 and 400 meters to obtain good values for the performance metrics. We use three values for the maximum transmission distance constraint in the next section to compare the new algorithm with other algorithms. These values are 100, 250 and 900 meters.

\section{2) Comparison Between the New Algorithm and Other Routing Algorithms}

In this section, we compare the new algorithm with the direct routing algorithm and the minimum transmission energy routing

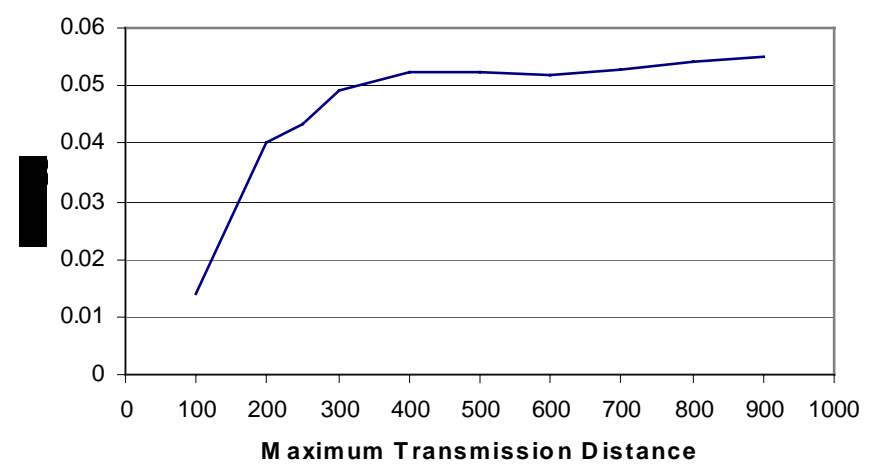

Fig. 3: Effect of the maximum transmission distance constraint on the average energy consumed per packet 


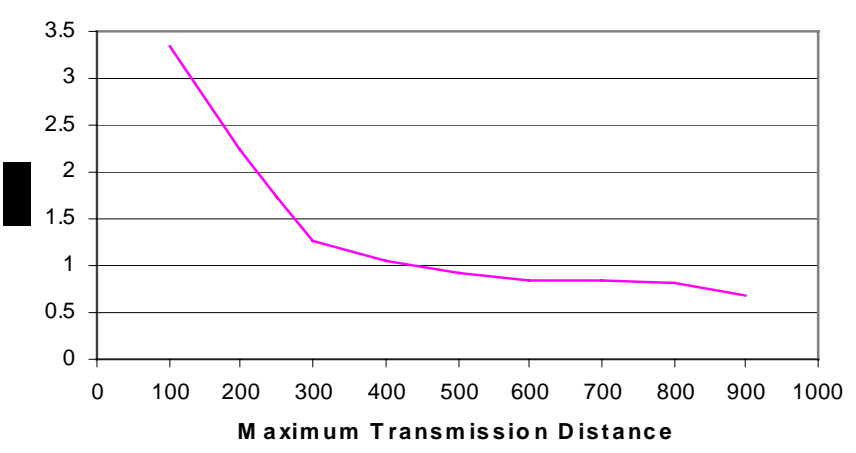

Fig. 4: Effect of the maximum transmission distance constraint on the average delay per packet

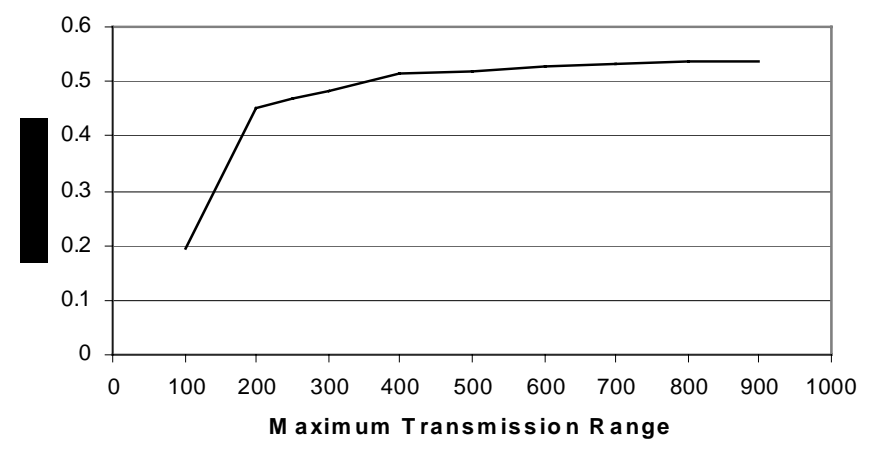

Fig. 5: Effect of the maximum transmission distance constraint on the total throughput

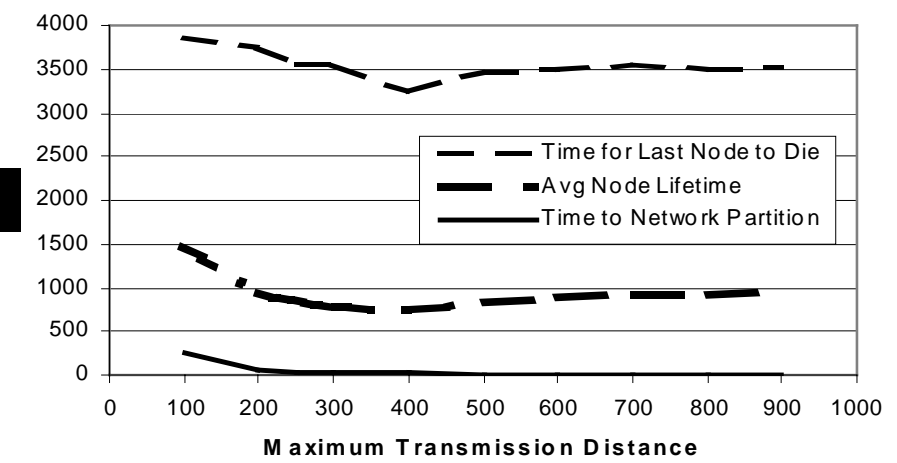

Fig. 6: Effect of the maximum transmission distance constraint on lifetime metrics

algorithm. We have three variants of the new algorithm corresponding to the three different values of the maximum transmission distance constraint. In the remaining figures, we call these algorithms 'New100', 'New250', and 'New900'.

Figures 7 through 12 show the performance comparison for the different metrics. By comparing the new algorithm performance when the maximum transmission distance constraint equals 100 , New100 in figures, with the minimum transmission energy routing algorithm, we can see that the new algorithm outperforms the minimum transmission energy routing algorithm in all metrics except in the throughput. For small values for the maximum transmission distance constraint, the network topology graph may not be strongly connected leading to loss of packets. This can be corrected by using an alternate route for transmitting data when a node has no neighbors due to the maximum transmission distance constraint.

By comparing the new algorithm performance when the maximum transmission distance constraint equals 900 , New 900 in figures, with the direct routing algorithm, we can see that both

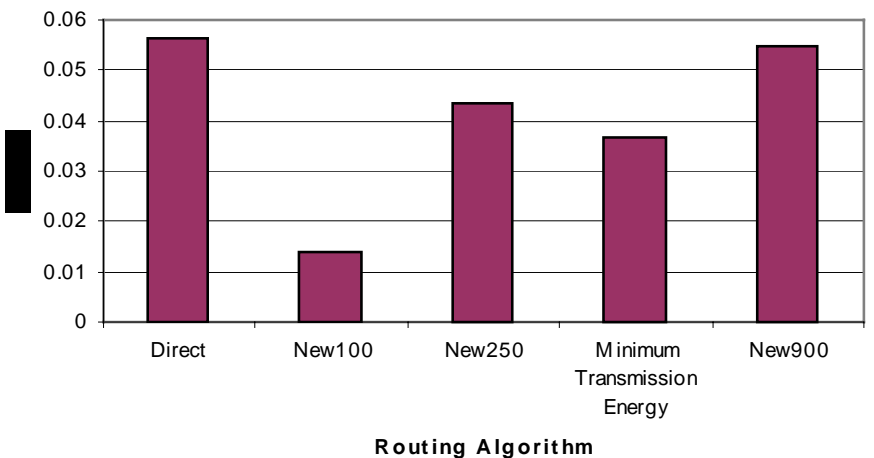

Fig. 7: Comparison between the new algorithm and other algorithms (average energy consumed per packet)

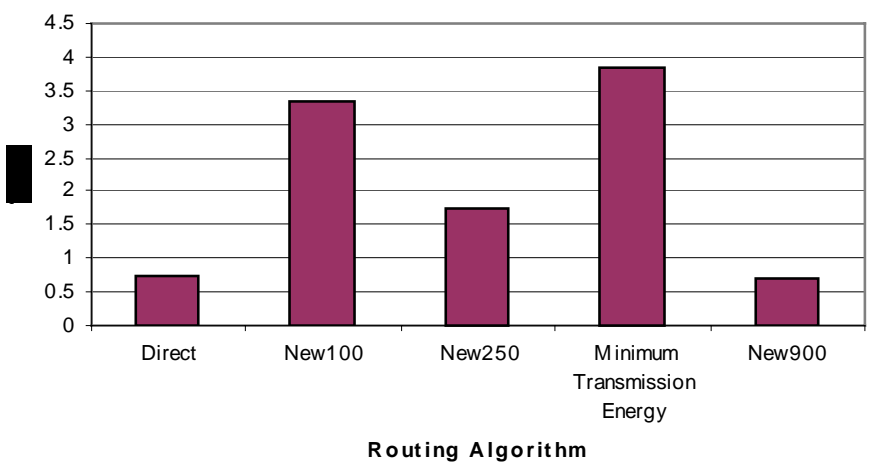

Fig. 8: Comparison between the new algorithm and other algorithms (average delay per packet)

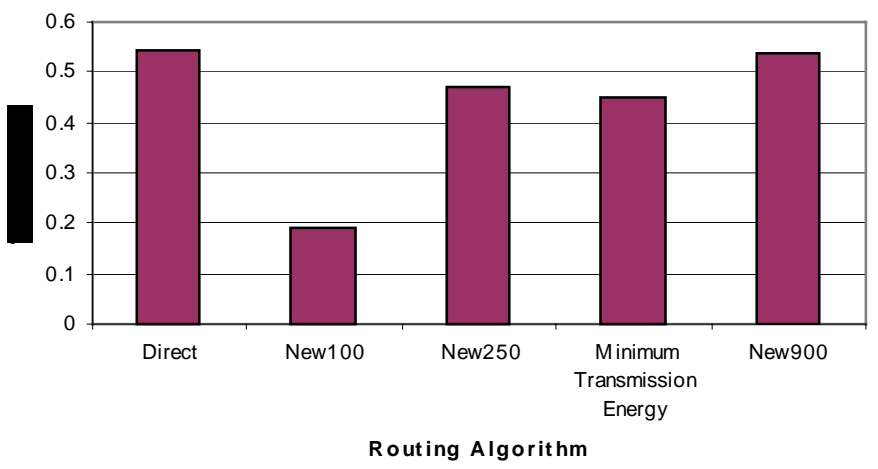

Fig. 9: Comparison between the new algorithm and other algorithms (total throughput)

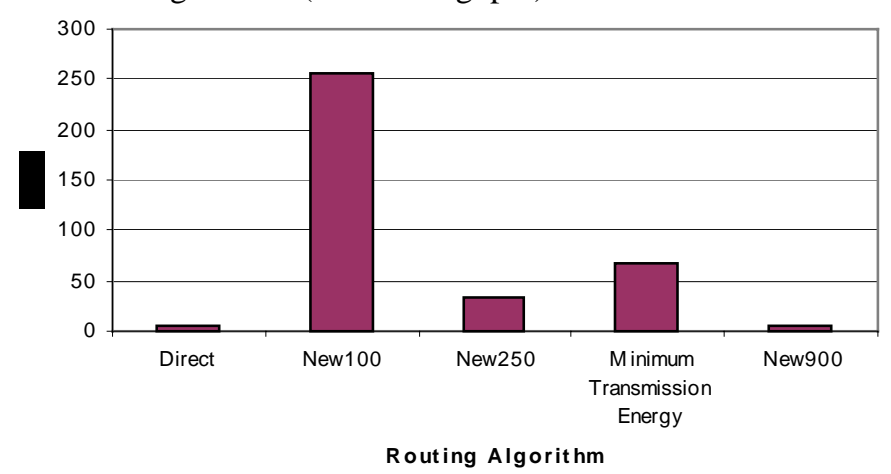

Fig. 10: Comparison between the new algorithm and other algorithms (time to network partition)

algorithms have approximately the same performance. For large values of the maximum transmission distance constraint, all nodes can reach the gateway directly and, hence, the minimum number of hops routing algorithm performance becomes similar to the direct routing algorithm. 


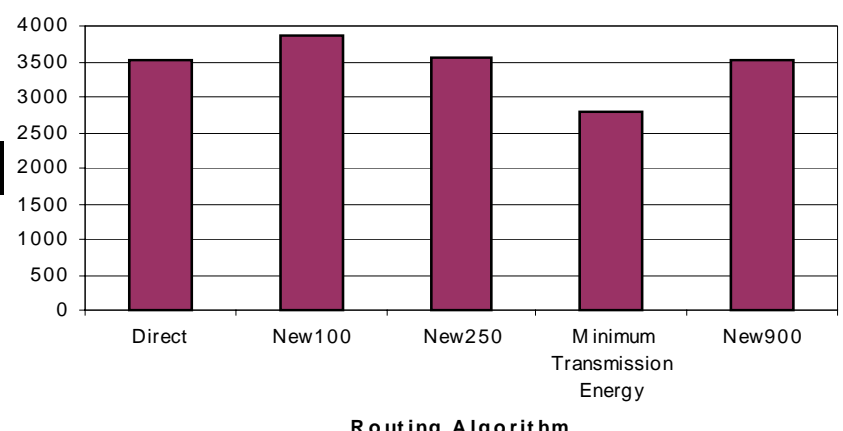

Fig. 11: Comparison between the new algorithm and other algorithms (time for last node to die)

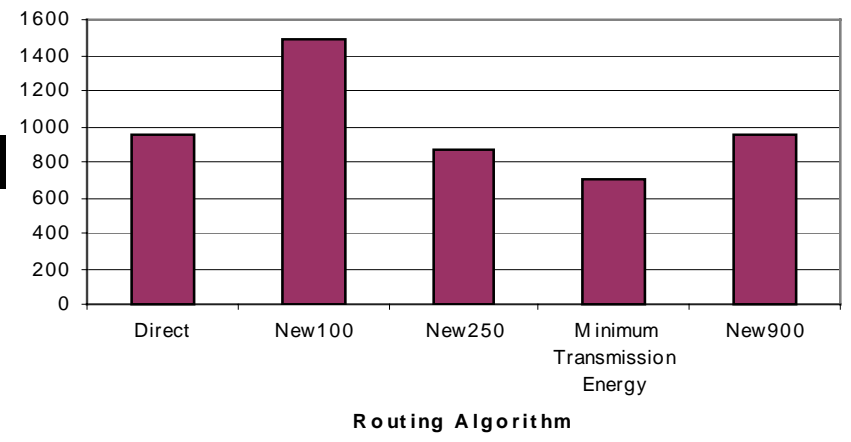

Fig. 12: Comparison between the new algorithm and other algorithms (average node lifetime)

The performance of the new algorithm when the maximum transmission distance constraint equals 250, New250 in figures, is acceptable under all performance metrics and presents a balance between the minimum transmission energy routing algorithm and the direct routing algorithm. Moreover, the New250 routing algorithm gives the best time for the last node to die. For a medium range value for the maximum transmission distance constraint, the new algorithm is able to choose small number of hops with a relatively short distance leading to good values for all performance metrics.

The choice of a value for the maximum transmission distance constraint depends on the required performance metrics and the nature of the sensor network mission.

\section{CONCLUSION AND FUTURE WORK}

In this paper, we presented a novel energy-aware routing algorithm that uses a constrained minimum number of hops routing algorithm. By constraining the maximum transmission distance, the new algorithm can achieve different performance objectives depending on the mission of the sensor network. For low values of the maximum transmission distance constraint, the network topology graph is sparse and the new algorithm performs like the minimum transmission energy routing algorithm. For large values of the maximum transmission distance constraint, the network topology graph is a complete graph and the minimum number of hops routing algorithm gives performance similar to the direct routing algorithm. For moderate values of the maximum transmission distance constraint, the performance of the new algorithm is acceptable under all performance metrics and presents a balance between the minimum transmission energy routing algorithm and the direct routing algorithm. For a moderate range value for the maximum transmission distance constraint, the new algorithm is able to choose small number of hops with a relatively short distance leading to good values for all performance metrics.
We plan to study the performance of the new algorithm under heavy load conditions. Under low values of the maximum transmission distance constraint, the network topology graph may not be strongly connected. Using alternate routes when a node has no neighbors is a direction for future research. Algorithms for clustering and inter-cluster interaction are also planned.

This paper gives a brief overview of some of features of the MAC protocol. Our pilot experiments show that the MAC protocol can increase the life of the network by an order of magnitude when combined with our routing approach. The detailed performance evaluation of the MAC protocol is the subject for a future paper.

\section{REFERENCES}

[1] P.J.M. Havinga, G.J.M. Smit, "Design techniques for low power systems", Jour. of Systems Architecture, vol. 46, no. 1, 2000.

[2] S. Singh, M. Woo and C. S. Raghavendra, "Power-aware routing in mobile ad hoc networks", ACM MOBICOM'98, Dallas, Texas, Oct. 1998.

[3] C-K. Toh, "Maximum battery life touting to support ubiquitous mobile computing in wireless ad hoc networks," IEEE Comm. Mag., Jun. 2001.

[4] W. Heinzelman, A. Chandrakasan, and H. Balakrishnan, "Energy-efficient communication protocols for wireless microsensor networks," HICSS '00, Jan. 2000.

[5] J.-H. Chang, L. Tassiulas, " Energy conserving routing in wireless ad-hoc networks, IEEE Infocom'00, 2000.

[6] W. Heinzelman, A. Sinha, A. Wang, and A. Chandrakasan, "Energy-scalable algorithms and protocols for wireless microsensor networks," Proc. ICASSP '00, Jun. 2000.

[7] V. Rodoplu and T. H. Meng, "Minimum energy mobile wireless networks", IEEE Jour. Selected Areas Comm., vol. 17, no. 8, pp. 1333-1344, Aug. 1999.

[8] M. Younis, M. Youssef and K. Arisha, "Energy-aware routing in cluster-based sensor networks," unpublished.

[9] M. Bhardwaj, T. Garnett, and A. P. Chandrakasan, "Upper bounds on the lifetime of sensor networks", In Proc. of ICC 2001, June 2001.

[10] Muir and J.J. Garcia-Luna-Aceves, "A Channel Access Protocol for Multihop Wireless Networks with Multiple Channels", Proc. IEEE ICC '98, Atlanta, Georgia, June 711, 1998.

[11]J.B. Andresen, T.S. Rappaport, and S. Yoshida, "Propagation measurements and models for wireless communications channels," IEEE Comm. Mag., vol. 33, no. 1, Jan. 1995.

[12] "Data sheet for the Acoustic Ballistic Module", SenTech Inc., http://www.sentech-acoustic.com/

[13] A. Sinha and A. P. Chandrakasan, "Energy aware software," Proc. of the 13th International Conference on VLSI Design, pp. 50-55, Calcutta, India. Jan. 2000.

[14] M. Bhardwaj, T. Garnett, and A. P. Chandrakasan, "Upper bounds on the lifetime of sensor networks", Proc. ICC 2001, Jun. 2001. 